\title{
Novel Incretin analogues improve autophagy and protect from mitochondrial stress induced by rotenone in SH-SY5Y cells
}

\author{
Jaishree Jalewa, Mohit Kumar Sharma and Christian Hölscher \\ Division of Biomedical and Life Sciences, Faculty of Health and Medicine, Lancaster \\ University, Lancaster, LA1 4YQ, UK.
}

\section{Accepted in the Journal of Neurochemistry}

Running title: Novel incretin analogues increase cell survival

\author{
Corresponding author: \\ Prof. Christian Hölscher, PhD \\ Lancaster University \\ Division of Biomedical and Life Sciences \\ Faculty of Health and Medicine \\ Furness college, \\ Lancaster, LA1 4YQ, UK \\ +44 (0) 1524594870 \\ Email: c.holscher@lancaster.ac.uk
}

\begin{abstract}
Abbreviations: Atg, autophagy related gene; Atg3, Autophagy-related protein 3; Bad, Bcl-2-associated death promoter; BBB, blood-brain barrier; Bcl-2, B-cell lymphoma 2; BrdU, 5-bromo-2-deoxyuridine; DMEM, Dulbecco's minimum essential medium; ETC, electron transport chain; GIP, glucose-dependent insulinotropic peptide; GLP-1, glucagon-like-peptide-1; GPCR, G-protein coupled receptor; IMM, inner mitochondrial membrane; IMS, inter membrane space; LDH, lactate dehydrogenase; OMM, outer mitochondrial membrane; PD, Parkinson's disease; PBS, phosphate buffered saline; PDH, pyruvate dehydrogenase; PI3K, phosphoinositide 3 kinase; SFM, serum free medium.
\end{abstract}




\begin{abstract}
Currently, there is no viable treatment available for Parkinson's disease (PD) that stops or reverses disease progression. Interestingly, studies testing the GLP-1 mimetic Exendin-4 have shown neuroprotective/neurorestorative properties in pre-clinical tests and in a pilot clinical study of PD. Incretin analogues were originally developed to treat type 2 diabetes and several are currently on the market. In this study, we tested novel incretin analogues on the dopaminergic SH-SY5Y neuroblastoma cells against a toxic mitochondrial complex I inhibitor, Rotenone. Here, we investigate for the first time the effects of six different incretin receptor agonists - Liraglutide, D-Ser2-Oxyntomodulin, a GLP-1/GIP Dual receptor agonist, dAla(2)-GIP-GluPal, Val(8)GLP-1-GluPal and exendin-4. Posttreatment with doses of 1,10 or $100 \mathrm{nM}$ of incretin analogues for $12 \mathrm{hrs}$ increased the survival of SH-SY5Y cells treated with $1 \mu \mathrm{M}$ Rotenone for $12 \mathrm{hrs}$. Furthermore, we studied the post-treatment effect of 100nM incretin analogues against $1 \mu \mathrm{M}$ Rotenone stress on apoptosis, mitochondrial stress, and autophagy markers. We found significant protective effects of the analogues against Rotenone stress on cell survival and on mitochondrial and autophagy-associated markers. The novel GLP-1/GIP Dual receptor agonist was superior and effective at a tenfold lower concentration compared to the other analogues. Using the Phosphatidylinositol 3-kinase (PI3K) inhibitor, LY294002, we further show that the neuroprotective effects are partially PI3K-independent. Our data suggest that the neuroprotective properties exhibited by incretin analogues against Rotenone stress involve enhanced autophagy, increased Akt-mediated cell survival and amelioration of mitochondrial dysfunction. These mechanisms can explain the neuroprotective effects of incretin analogues reported in clinical trials.
\end{abstract}

Keywords: Parkinson's disease, incretins, autophagy, growth factors, oxidative stress, rotenone 


\section{Introduction}

Parkinson's disease (PD) is the second common multifactorial and clinically heterogenous neurodegenerative movement disorder, distinguished by the loss of dopaminergic neurons in the substantia nigra pars compacta $(\mathrm{SNpc})$. The aetiology of the disease is still unclear, but the three key mechanisms involved in this disease are alphasynuclein aggregation, mitochondrial dysfunction and oxidative stress (Schapira \& Jenner 2011, Alberio et al. 2012). The critical symptoms of the disease are tremor at rest, bradykinesia and rigidity followed by late-stage symptoms of gait dysfunction and postural instability (Obeso et al. 2010). Despite good progress since the clinical identification of PD, no viable treatment exists yet that could halt or reverse disease progression (Shulman et al. 2011). Research in the last decade has highlighted abnormal glucose metabolism as a risk factor for PD and potential of anti-diabetic drugs in treating neurodegenerative disorders (Hu et al. 2007, Holscher 2012, Aviles-Olmos et al. 2013, Bassil et al. 2014, Holscher 2014b, Sharma et al. 2014). Exendin-4 (Byetta) is a GLP-1 receptor agonist. One study showed that Exendin-4 promotes adult neurogenesis in vitro and in vivo, improves dopamine imbalance and increases dopaminergic neurons in the SN (Bertilsson et al. 2008). Another study demonstrated that GLP-1 receptor stimulation reverses established nigral lesions in 6-hydroxydopamine (6-OHDA) and lipopolysaccaride (LPS) models of PD (Harkavyi et al. 2008). Further studies also show that Exendin-4 protects dopaminergic neurons (Kim et al. 2009, Li et al. 2009). Importantly, a pilot study in Parkinson's patients showed good neuroprotective effects with exendin-4 (Aviles-Olmos et al. 2013, 2014). A larger phase II trial is currently ongoing.

Incretins are hormones that activate insulin secretion after meal ingestion in a glucosedependent manner. Glucose-dependent insulinotropic polypeptide (GIP) and glucagonlike peptide-1 (GLP-1) are key members of this family (Campbell \& Drucker 2013). Incretins cross the blood brain barrier and activate neurogenesis, synaptogenesis and growth factor signalling in neurons. A range of novel GLP-1 and GIP agonists resistant to 
protease cleavage have been developed, which exhibit extended half-life and superior receptor activation properties (Holscher 2012, Holscher 2014a). Liraglutide (Victoza ${ }^{\circledR}$ ) is a GLP-1 mimetic, a modified analogue of human GLP-1 (Cho et al. 2014, Manandhar \& Ahn 2015). We have previously reported very promising effect of Liraglutide on SHSY5Y cells against methyl glyoxal oxidative stress (Sharma et al. 2014). Liraglutide also showed good effects in animal models of Alzheimer's disease (AD) (McClean et al. 2011, McClean \& Holscher 2014) and in a pilot clinical trial in Alzheimer's disease (AD) (Gejl et la., 2016). A phase II clinical trial in patients with AD is ongoing (Holscher 2014b). Liraglutide and other GLP-1 mimetics showed good protective effects in the 1methyl-4-phenyl-1,2,3,6-tetrahydropyridine (MPTP) mouse model of PD (Liu et al., 2015b). Val(8)GLP-1-GluPal is a new GLP-1 analogue that has longer biological half-life (Lennox et al. 2013). Our recent research has demonstrated neuroprotective effects of Val(8)GLP-1-GluPal in the MPTP PD mouse model (Zhang et al. 2015). Oxyntomodulin is a GLP-1 and glucagon receptor dual agonist (Pocai 2012). Our research showed that the protease-resistant analogue D-Ser2-Oxyntomodulin showed protective effects from MPTP-induced motor impairment, improved dopamine synthesis and protected synapses (Liu et al. 2015b). In addition to this, newer dual agonists have been developed that activates both GLP-1 and GIP receptors (Finan et al. 2013). A recent study exhibited the protective role of GLP-1/GIP Dual agonists in the MPTP PD model where the drug reduced/reversed most of the PTP-induced motor impairments, increased the number of tyrosine hydroxylase $(\mathrm{TH})$ positive neurons, activated the cytoprotective kinase Akt, and interestingly, enhanced the levels of the neuroprotective brain derived neurotropic factor (BDNF) (Ji et al. 2015). A second study found that this dual agonist reduced chronic inflammation in the brain and protected against the loss of synapses (Cao et al. 2016). The sister incretin GIP also shows promising protective effects. dAla2-GIP-GluPal is a stable, long-acting potent GIP analogue (Martin et al. 2013). Our research demonstrated that dAla-2GIP-GluPal treatment improved the locomotor and exploratory activity of MPTP mice, restored TH positive dopaminergic neurons, reduced the chronic inflammation response and decreased $\mathrm{Bax} / \mathrm{Bcl}-2$ ratio (Li et al. 2016).

In this study, we investigated the protective effects of different incretin analogues Liraglutide, D-Ser2-Oxyntomodulin, a GLP-1/GIP Dual agonist with a C16 fatty acid 
attached, dAla(2)-GIP-GluPal, Val(8)GLP-1-GluPal and Exendin-4 on apoptosis, mitochondrial stress, and autophagy induced by Rotenone.

\section{Materials and Methods}

\subsection{Materials}

Cell proliferation kit II (2,3- Bis(2-methoxy-4-nitro-5-sulfophenyl)-2H-tetrazolium-5carboxanilide (XTT)), Cytotoxicity Detection KitPLUS (Lactate dehydrogenase (LDH)) and cell proliferation ELISA 5-bromo-2-2deoxyuridine (BrdU) (colorimetric) kit were purchased from Roche Diagnostics Limited (West Sussex, UK). The Mitochondrial Marker Antibody Sampler Kit, Pro-Survival Bcl-2 Family Antibody Sampler Kit, ProApoptosis Bcl-2 Family Antibody Sampler Kit, Autophagy Antibody Sampler Kit, Loading Control Antibody Sampler Kit and phospho-Akt (Ser473) (D9E), Akt (pan) (C67E7) and anti-rabbit IgG, HRP-linked secondary antibody were purchased from Cell signaling technology (New England Biolabs (UK) Ltd, Hertfordshire, UK). Cell lysis buffer was obtained from Cell signaling technology, Quick start protein assay reagent from BIO-RAD Laboratories Ltd (Hertfordshire, UK), Amersham ECL Prime western blotting detection reagent from GE, Healthcare Life Sciences (Buckinghamshire, UK). XCell4 SureLock ${ }^{\mathrm{TM}}$ Midi-Cell Electrophoresis System, iBlot ${ }^{\circledR} 2$ Gel Transfer Device, iBlot ${ }^{2} 2$ Transfer Stacks, PVDF, regular size $\left(\right.$ Novex $\left.^{\mathrm{TM}}\right)$, pre-cast polyacrylamide NuPAGE® Novex ${ }^{\circledR}$ 4-12\% Bis-Tris Midi Gels and Restore ${ }^{\mathrm{TM}}$ Western Blot Stripping Buffer were purchased from Life technologies. Other materials for western blotting and cell culture were obtained from Invitrogen. No ethical approval was required for this work.

\subsection{Peptides}

Incretins were purchased from Chinapeptides and GL Biochem (Shanghai) Ltd. The purity of each peptide was analysed by reversed-phase HPLC and characterised using matrix-assisted laser desorption/ionization time of flight (MALDI-TOF) mass spectrometry, with a purity $>99 \%$. Peptides were reconstituted in ultrapure water (MilliQ) to a concentration of $1 \mathrm{mg} / \mathrm{ml}$ and aliquots prepared and stored at $-20^{\circ} \mathrm{C}$. 


\section{Peptides tested and AA sequences:}

Exendin-4

HGEGTFTSDLSKQMEEEAVRLFIEWLKNGGPSSGAPPPS

Liraglutide

HAEGTFTSDVSSYLEGQAA[Lys-yE-C ${ }_{16}$ acyl]EFIAWLVRGRG

Val-8-GLP-1-GluPal (Lennox et al. 2013)

HVEGTFTSDVSSYLEGQAA[Lys-yE-C ${ }_{16}$ acyl]EFIAWLVRGRG

dAla-2GIP-GluPal (Faivre \& Holscher 2013)

YdAEGTFISDYSIAMDKIHQQDFVNWLLAQKGKKNDW[Lys-yE-C ${ }_{16}$ acyl]HNITQ

GLP-1/GIP dual agonist (Cao et al., 2016)

YXEGTFTSDYSIYLDKQAAXEFVNWLLAGGPSSGAPPPSK-NH2

$\mathrm{X}=$ aminoisobutyric acid; $\mathbf{K}=$ Lys $\mathrm{C}_{16}$ acyl

D-Ser2-oxyntomodulin (Liu et al. 2015a)

H(d-S)QGTFTSDYSKYLDSRRAQDFVQWLMNTKRNKNNIA

\subsection{Cell culture}

The human neuroblastoma cell line SH-SY5Y is a thrice cloned subline of the neuroblastoma cell line SK-N-SH, established in 1970 from a metastatic bone tumour. The SH-SY5Y cells were purchased from LGC standards (Middlesex, UK) (ATCC No. CRL-2266), and maintained in Dulbecco's minimum essential medium, DMEM+F12 (1:1) GlutaMax supplemented with 10\% heat-inactivated foetal bovine serum (FBS) and 100 Units $/ \mathrm{ml}$ of Penicillin and $100 \mu \mathrm{g} / \mathrm{ml}$ of Streptomycin at $37^{\circ} \mathrm{C}$ in a humidified incubator with $95 \%$ air and $5 \% \mathrm{CO}_{2}$. The cells were sub-cultured at $80 \%$ confluency and seeded at 1:8 ratio. Live cells were counted for next passage and seeded desired number of cells for the assays by using Countess ${ }^{\mathrm{TM}}$ Automated Cell Counter (Invitrogen, UK). 
Countess is based on the usual trypan blue dye exclusion principle where dead cells are permeable to dye and stains blue while viable cells do not take up the impermeable dye. Media of the cells was changed every 3-4 days.

\subsection{Measurement of cell viability, cytotoxicity and proliferation}

Cell viability, cytotoxicity, and proliferation were estimated using Cell proliferation kit II (XTT), Cytotoxicity Detection KitPLUS kit (LDH) and Cell Proliferation ELISA, BrdU (colorimetric) kit, respectively. The assays were formatted in Thermo Scientific ${ }^{\mathrm{TM}}$ Nunc $^{\text {TM MicroWell }}{ }^{\text {TM }}$ 96-well plates. SH-SY5Y cells were seeded at a density of 10,000 cells/well for $24 \mathrm{~h}$. Thereafter, the cells were serum starved for $12 \mathrm{~h}$ in serum free medium (SFM) and were stressed with different concentration of Rotenone $(0.1,1$ and $10 \mu \mathrm{M})$ for $12 \mathrm{~h}$ followed by post-treatment with different concentrations $(1,10,100 \mathrm{nM})$ of incretin analogues for $12 \mathrm{~h}$. Supernatant was removed (for LDH assay) and $5 \mathrm{ml}$ of XTT labelling reagent and $0.1 \mathrm{ml}$ of electron-coupling reagent diluted 1:1 with SFM was added per microplate followed by incubation at $37^{\circ} \mathrm{C}$ for $4 \mathrm{~h}$. XTT assay involves cleavage of the yellow tetrazolium salt XTT to form an orange coloured formazan dye by metabolically active cells (mitochondrial dehydrogenase activity). So, the cell viability is spectrophotometrically quantified based on the formation of this soluble formazan product, which is directly proportional to the number of living cells in the sample. The plate was gently shaken for 5 min on Microtitre plate shaker (Stuart, Staffordshire, UK) and absorbance measured at $492 \mathrm{~nm}$ and $690 \mathrm{~nm}$ (reference wavelength) in a Infinite 200 PRO microplate reader (Tecan, Reading, UK).

Using Cell Proliferation ELISA, BrdU (colorimetric) kit, the cell proliferation was evaluated in actively proliferating SH-SY5Y cells based on the incorporation of pyrimidine analogue $\mathrm{BrdU}$ instead of thymidine during DNA synthesis. After $12 \mathrm{hrs}$ of post-treatment, BrdU labeling reagent was added and the plate was incubated at $37^{\circ} \mathrm{C}$ for $4 \mathrm{hr}$. BrdU incorporation was measured as per the manufacturer's assay procedure and the plate was read at 450nm wavelength (reference wavelength 690nm) using Infinite 200 PRO microplate reader.

Cytotoxicity Detection KitPLUS (LDH) kit is a colorimetric assay that quantitatively 
measures the LDH activity, directly proportional to the cytotoxicity. $\mathrm{LDH}$ is a stable cytosolic enzyme that is released upon cell lysis, which results in the formation of a red formazan product and the amount of colour formed is proportional to the number of lysed cells. The assay was conducted according to the manufacturer's instructions and absorbance measured at 492nm and 690nm (reference wavelength).

\subsection{Western blotting}

$10^{6}$ cells were grown in $100 \mathrm{~mm}$ plates and after serum starvation for $12 \mathrm{hrs}$, cells were stressed with $1 \mu \mathrm{M}$ Rotenone for $12 \mathrm{hrs}$ and post-treated with $100 \mathrm{nM}$ incretin analogues for further $12 \mathrm{hrs}$. Cells were then washed with cold 1X PBS buffer followed by addition of $1 \mathrm{X}$ cell lysis buffer containing protease inhibitor. After two freeze thaw cycles, the lysate was collected and total protein was extracted by centrifugation at 13,000rpm for 20min. Quick start Bradford protein assay reagent was used to estimate the protein concentration. This assay is based on the principle of Bradford method, which involves Commasie Brilliant Blue G-250 dye that binds to the proteins and gets converted to stable unprotonated blue form. The Bovine Serum Albumin standards included set of 7 concentrations of $\operatorname{BSA}(2,1.5,1,0.75,0.5,0.25,0.125 \mathrm{mg} / \mathrm{ml})$, used to prepare standard curve. $5 \mu 1$ of the diluted sample was added into a clear bottom 96-well plate in quadruplicate along with $20 \mu 1$ of double distilled water and $150 \mu 1$ of the Quick start protein assay reagent and the plate read after $5 \mathrm{~min}$ at $595 \mathrm{~nm}$ wavelength in a Infinite 200 PRO microplate reader (Tecan, Reading, UK). Cell lysate containing $3 \mu \mathrm{g}$ of protein was separated on 4-12\% gradient Bis-Tris gel with Novex pre-stained marker and electrophoresed in running buffer at $180 \mathrm{mV}$ for $70 \mathrm{~min}$ followed by transfer to polyvinylidene difluoride (PVDF) membrane using iBlot@ 2 Gel Transfer Device. Following protein transfer, the membrane was washed in 1X TBS-T (tris-buffered saline with $0.05 \%$ Tween-20, $\mathrm{pH} 8$ ) and blocked in $5 \%$ skimmed milk for $1 \mathrm{hr}$ at $25^{\circ} \mathrm{C}$. The membrane was then incubated with anti-pAkt (Ser473) (1:1000) and other primary antibodies at $4^{\circ} \mathrm{C}$ overnight and after three washes (5min each) in TBS-T further incubated with 1:2000 horseradish peroxidase-conjugated anti-rabbit IgG. All the primary antibodies used were generated in rabbit unless specified. The protein bands were visualized by Amersham ECL Prime western blotting detection reagent according to the 
manufacturer's instructions. ChemiDoc MP Imaging System with Image Lab software (BIO-RAD) was used to image chemiluminescent bands and perform the analysis of each band intensity. Image $\mathrm{Lab}^{\mathrm{TM}}$ software controls image capture and optimiszation and produces reports. Beta-actin was used as loading control and relative peak intensity of each marker band analysed on Mac Numbers after normalizing with loading control. To reprobe, the membranes were incubated with Restore stripping buffer (Thermo Scientific, UK) with some agitation for $15 \mathrm{~min}$ at RT followed by 3 washes in TBS-T for $5 \mathrm{~min}$ each. The membranes were checked with chemiluminescent detection preceding incubation in another primary antibody of interest.

\subsection{Statistical analysis}

Statistical analysis was performed using Prism 6 for Mac OS X (GraphPad software Inc., La Jolla, CA, USA) with the $95 \%$ level of probability and the results were presented as mean \pm SEM. Data were analysed by one-way ANOVA, followed by Bonferroni's multiple comparison post-hoc test.

\section{Results}

\section{1 $1 \mu \mathrm{M}$ Rotenone stress for $12 \mathrm{hrs}$ increases LDH levels and decreases cell viability}

The stress duration of $12 \mathrm{hrs}$ with rotenone was selected in initial tests as to be most effective. In order to standardise the Rotenone dose, cells were stressed with different concentrations of Rotenone $(0.1,1,10 \mu \mathrm{M})$ for $12 \mathrm{hrs}$ and cell viability was determined after 12hrs of DMEM (Glutamax) medium post-treatment in order to keep the assay format identical to the drug analogue testing experiments to be performed later. In the XTT assay, we found that there was a dose-dependent decrease in the cell viability (\% control) at $0.1,1$ and $10 \mu \mathrm{M}$ rotenone concentration with $28.7 \%$ ( $<<0.0001$ ), $32.5 \%$ $(\mathrm{p}<0.0001)$ and $36.5 \%(\mathrm{p}<0.0001)$ percentage decrease, respectively. The percentage increase in LDH levels (\% control) was 40\% ( $<<0.05), 88.2 \%$ ( $<<0.0001$ ) and $24.7 \%$ with $0.1,1$ and $10 \mu \mathrm{M}$ rotenone exposure. The $\mathrm{LDH}$ levels were significantly $(\mathrm{p}<0.05)$ higher at $1 \mu \mathrm{M}$ rotenone when compared to the $0.1 \mu \mathrm{M}$ rotenone stressed cells. The percentage 
increase in cell proliferation levels (\% control) were 26.4\% ( $\mathrm{p}<0.01), 27.7 \%(\mathrm{p}<0.01)$ and $5.6 \%$ with $0.1,1$ and $10 \mu \mathrm{M}$ rotenone stress. For the further studies, we chose $1 \mu \mathrm{M}$ concentration of rotenone stress as it induced highly significant effect at LDH levels ( $88.2 \%$ increase) and cell viability (32.5\% decrease), both shown by LDH and XTT assays, respectively (Fig. 1).

\subsection{Post-treatment with Incretin analogues increases cell survival and reduces cytotoxicity}

To determine the protective effect of incretin analogues: Liraglutide (Lira), $d$-Ser2Oxyntomodulin (Oxm), GLP-1/GIP Dual agonist (Dual), D-Ala²-GIP-GluPal, Val(8)GLP-1-GluPal and Exendin-4 in the SH-SY5Y human neuroblastoma cells against Rotenone toxicity, XTT and LDH assays were performed. All six analogues were tested at three different concentrations $(1,10$ and $100 \mathrm{nM})$ against $1 \mu \mathrm{M}$ Rotenone pre-stress. All assays were formatted for $12 \mathrm{hrs}$ pre-stress followed by $12 \mathrm{hrs}$ post-treatment. Control cells were grown for $12 \mathrm{hrs}$ in DMEM (Glutamax) medium (without stress) followed by further $12 \mathrm{hrs}$ in medium only (no drug).

\subsubsection{Post-treatment with 1nM incretin analogues}

There is $36 \%$ decrease in the cell viability at $1 \mu \mathrm{M}$ Rotenone when compared to the control cells ( $\mathrm{p}<0.0001$ ). 1nM Lira, Oxm and Val(8)GLP-1-GluPal post-treatment increases the cell viability significantly $(\mathrm{p}<0.01)$. However, $1 \mathrm{nM}$ Dual enhances the cell viability most $(\mathrm{p}<0.0001)$ under these experimental conditions $(\mathrm{p}<0.0001)$ (Fig 1c). Lira

$(\mathrm{p}<0.0001), \operatorname{Val}(8)$ GLP-1-GluPal $(\mathrm{p}<0.05)$ and Exendin-4 $(\mathrm{p}<0.0001)$ exhibit significant decrease in the LDH levels. Lira seems to perform better in lowering the cytotoxicity as compared to Oxm $(\mathrm{p}<0.01)$ and D-Ala ${ }^{2}-G I P-G l u P a l(p<0.01)$. Exendin-4 ameliorates LDH levels when compared to Oxm and D-Ala ${ }^{2}-$ GIP-GluPal (p<0.001) (Fig 1d). 


\subsubsection{Post-treatment with 10nM incretin analogues}

$1 \mu \mathrm{M}$ Rotenone exposure results in $33.3 \%$ decrease in the cell viability when compared to the control cells (p<0.0001) (Fig 2a). 10nM Lira, Oxm, Dual and Val(8)GLP-1-GluPal exhibits highly significant increase in the cell viability. D-Ala'2-GIP-GluPal and Exendin4 did not show significant increase in the cell viability at this concentration (Fig 2a), which is in line with the 1nM concentration (Fig 1d). Lira, Oxm and Val(8)GLP-1-GluPal performs better than Exendin-4 $(\mathrm{p}<0.05)$ in improving cell viability at 10nM dosage. Lira $(\mathrm{p}<0.01), \operatorname{Val}(8)$ GLP-1-GluPal $(\mathrm{p}<0.001)$ and Exendin-4 $(\mathrm{p}<0.0001)$ significantly reduces the LDH levels (Fig 2b). 10nM Exendin-4 demonstrates more potential in reducing the cytotoxicity when compared to Oxm $(\mathrm{p}<0.01)$ and D-Ala ${ }^{2}-G I P-G l u P a l$ $(\mathrm{p}<0.05)$.

\subsubsection{Post-treatment with 100nM incretin analogues}

Cell viability decreases by $31 \%$ on exposure to $1 \mu \mathrm{M}$ Rotenone stress for $12 \mathrm{hrs}$, when compared to the control conditions ( $\mathrm{p}<0.0001)$. 100nM Lira, Oxm, Dual and D-Ala²-GIPGluPal demonstrates dose dependent behaviour in increasing the cell viability (Fig 2c) (when compared to Fig 1d and 2a, 1nM and 10nM dose, respectively). Although D-Ala ${ }^{2}-$ GIP-GluPal do not show significant effects at 1 and 10nM doses but did induce a highly significant increase in the cell viability at 100nM dosage. Val(8)GLP-1-GluPal and Exendin-4 do not show significant increase in the cell viability. It appears that Val(8)GLP-1-GluPal works better at lower doses (Fig 1d, 2a) when compared to 100nM dosage. Lira, Oxm and Dual increases cell survival at all three concentrations. Exendin-4 did not increase the cell viability at any of these concentrations. In the LDH assay, 100nM Lira (p<0.01), Oxm (p<0.001), Val(8)GLP-1-GluPal (p<0.0001) and Exendin-4 $(\mathrm{p}<0.0001)$ decreases the cytotoxicity. Oxm ( $<<0.05)$, Val(8)GLP-1-GluPal $(\mathrm{p}<0.001)$ and Exendin-4 $(\mathrm{p}<0.001)$ decreases LDH levels much more as compared to the D-Ala ${ }^{2}-$ GIP-GluPal (Fig 2d). At 100nM dose, Val(8)GLP-1-GluPal (p<0.001) and Exendin-4 $(\mathrm{p}<0.001)$ performs better than the Dual agonist with respect to lowering the cytotoxicity. Exendin-4 decreases the LDH levels significantly at all three concentrations. 


\subsection{Post-treatment with incretin analogues activates cell survival mechanisms}

$12 \mathrm{hrs}$ post-treatment with $100 \mathrm{nM}$ incretin analogues against $1 \mu \mathrm{M}$ Rotenone pre-stress on survival marker proteins. There is a significant increase in pAkt (Ser473)/panAkt expression in the incretin post-treated groups compared to the control. Lira (L) showed an increase of $146 \%(\mathrm{p}<0.05)$ and $16.5 \%$ increase in pAkt (Ser473)/panAkt expression when compared to the control cells and rotenone stressed cells, respectively. Oxm (O) shows and increase of $132.7 \%$ ( $\mathrm{p}<0.05)$ compared to control cells. Dual Agonist (D), Val(8)GLP-1-GluPal (V) and Exendin-4 (E) post-treatment results in almost 3-fold increase ( $\mathrm{p}<0.001)$ in the pAkt (Ser473)/panAkt ratio when compared to control cells. DAla(2)-GIP-GluPal (A) post-treatment demonstrates a highly significant 3.5-fold increase $(\mathrm{p}<0.0001)$ when compared to the control cells and a significant $(\mathrm{p}<0.05) 63 \%$ increase as compared to the Rotenone stressed cells (Fig. 3a).

To further elucidate the downstream Akt survival mechanism, we quantified protein levels of the phosphorylated forms of Bcl-2 and BAD, phospho-Bcl-2 (Ser70) and phospho-BAD (Ser112), respectively. Bcl-2, B-cell lymphoma 2 is the key anti-apoptotic marker and its phosphorylation at several sites including Ser70 is required in response to the apoptotic stimuli to protect the cell from dying. We found a significant increase in phospho-Bcl-2 (Ser70) expression in the incretin post-treated cells as compared to the control cells (Fig. 3b). Lira (L) post-treatment shows an impressive 6-fold increase $(\mathrm{p}<0.0001)$ in the phospho-Bcl-2(Ser70) expression levels when compared to the control and a $29 \%$ increase as compared to the untreated Rotenone stressed cells. Oxm (O) $(\mathrm{p}<0.001)$ as well as D-Ala²-GIP-GluPal (A) $(\mathrm{p}<0.0001)$ shows a highly significant approximately 5 -fold increase in phospho-Bcl-2 (Ser70) expression when compared to the control cells. Dual (D) results in a 5.8-fold $(\mathrm{p}<0.0001)$ and a $24.8 \%$ increase in phospho-Bcl-2 (Ser70) expression when compared to the control and Rotenone stressed cells, respectively. Val(8)GLP-1-GluPal (V) and Exendin-4 (E) post-treatment results in almost 4.8-fold $(\mathrm{p}<0.001)$ and 4.4-fold increase $(\mathrm{p}<0.01)$, respectively, in the phosphoBcl-2 (Ser70) expression when compared to the control cells (Fig. 3b). 
Furthermore, we observed an increase in phospho-BAD (Ser112) expression in the stressed cells post-treated with incretin analogues (Fig 3c). BAD is a pro-apoptotic member of the Bcl-2 family but it becomes inactive when phosphorylated by different survival factors, thus phosphorylation of BAD at site Ser112 promotes cell survival. Lira (L) and Oxm (O) post-treatment results in an increase of $20 \%$ in the phospho-BAD (Ser112) expression when compared to the untreated Rotenone stressed cells. Dual (D) shows a significant $(\mathrm{p}<0.01) 1.8$-fold increase in the phospho-BAD (Ser112) expression compared to the control. D-Ala'a -GIP-GluPal (A) as well as Val(8)GLP-1-GluPal (V) post-treatment exhibits a highly significant 2 -fold increase $(\mathrm{p}<0.001)$ in the phosphoBAD (Ser112) expression compared to the control and a significant $51 \%$ increase $(\mathrm{p}<0.05)$ when compared to the untreated Rotenone stressed cells. Exenatide (E) posttreatment results in almost 1.8-fold ( $\mathrm{p}<0.01)$ increase in the phospho-BAD (Ser112) expression compared to the control cells (Fig. 3c).

\subsection{Influence of Incretin analogues on Rotenone - induced mitochondrial damage in the neuroblastoma cells.}

Mitochondrial protein PDHA1, also known as C54G1, is an alpha subunit of pyruvate dehydrogenase. Pyruvate dehydrogenase (C54G1) monoclonal antibody detects endogenous levels of total pyruvate dehydrogenase $\alpha 1$ subunit. In the current experimental setting, we found significant $(\mathrm{p}<0.0001)$ increase of $71 \%$ in the expression of pyruvate dehydrogenase in the stressed state. Lira (L), Oxm (O), Dual (D), D-Ala ${ }^{2}-$ GIP-GluPal (A), Val(8)GLP-1-GluPal (V), and Exenatide (E) post-treatment exhibits an increase of $61.5 \%$ ( $\mathrm{p}<0.0001), 76.2 \%$ ( $\mathrm{p}<0.0001), 68.5 \%$ ( $<<0.0001), 53.2 \%(\mathrm{p}<0.0001)$, $57 \%$ ( $<<0.0001), 45.3 \%(\mathrm{p}<0.001)$ respectively, in pyruvate dehydrogenase expression when compared to the control condition (Fig 4a). Oxm (O) shows an increase of 3\% in the expression of pyruvate dehydrogenase when compared to the stressed state (Fig 4a).

\subsection{Incretin post-treatment enhances autophagy, a plausible mechanism to protect}




\section{SH-SY5Y cells from Rotenone - induced mitochondrial oxidative dysfunction.}

We observed a 26.5\% $(\mathrm{p}<0.05)$ increase in Atg7 expression in the rotenone stressed cells compared to the control (Fig 4b). Lira (L), Oxm (O), Dual (D), D-Ala²-GIP-GluPal (A), Val(8)GLP-1-GluPal (V), and Exendin-4 (E) post-treatment shows an increase of 29.3\% $(\mathrm{p}<0.01), 30 \%(\mathrm{p}<0.01), 13.5 \%, 35.7 \% \quad(\mathrm{p}<0.001), 23.8 \% \quad(\mathrm{p}<0.05)$, and $19.2 \%$, respectively in the Atg7 expression when compared to the control condition.

In addition, we observed a $47.7 \%$ increase in the Atg 3 expression in the rotenone stressed cells compared to the control. Lira $(\mathrm{L})$ and Oxm $(\mathrm{O})$ treatment results in $39.5 \%$ and 22.5\% increase in Atg3 expression, respectively, when compared to the control. Autophagosomal marker protein LC3 (light chain 3) is another principal autophagyassociated marker (Johansen \& Lamark 2011). We found a 43.4\% increase in the LC3A/B expression in the rotenone stressed cells compared to the control. Lira (L) posttreatment induced a $34.5 \%$ increase in $\mathrm{LC} 3 \mathrm{~A} / \mathrm{B}$ expression when compared to the control. These results indicate the role of incretin analogues in activation of autophagy as a possible protective mechanism. Lira (L) post-treatment exhibited an increase in all the autophagy-associated markers studied. D-Ala'-GIP-GluPal (A) treatment increased Atg7 expression by $35.7 \%$ ( $\mathrm{p}<0.001)$, compared to control.

\subsection{Incretin analogues ameliorate the inhibitory effect of PI3K inhibitor pre- exposure on autophagy and cell survival.}

To confirm whether the Akt activation by incretin analogues is mediated via PI3K and to study the mechanism underlying the protection exhibited by these incretin analogues, we

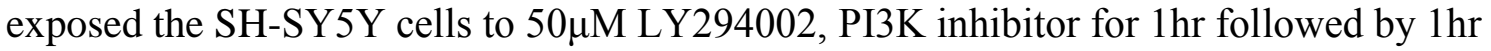

of incretin treatment. Afterwards cells were harvested and cell lysate prepared for western blot analysis. We examined Atg3 levels and found that in the presence of inhibitor, there is a significant $31.5 \%$ decrease $(\mathrm{p}<0.01)$ in the expression levels of Atg3, when compared to the control. Post-treatment with Lira (L), Oxm (O), Dual (D), D-Ala²-GIP-GluPal (A) and $\mathrm{Val}(8)$ GLP-1-GluPal (V) shows an increase of 1.8-fold ( $\mathrm{p}<0.0001)$, 1.9-fold 
( $\mathrm{p}<0.0001), 1.8$-fold $(\mathrm{p}<0.0001), 1.6$-fold $(\mathrm{p}<0.001)$ and 1.3-fold, respectively in the Atg3 expression when compared to the stressed condition (Fig 5a). Exendin-4 (E) posttreatment did not ameliorate the Atg3 decrease in the stressed state. In addition, we examined the pBcl-2 levels and found that LY294002 exposure decreased the pBcl-2 levels by $27 \%$ (Fig 5b). Interestingly, post-treatment with incretin analogues improved the pBcl-2 expression levels. Lira (L), Oxm (O), Dual (D), D-Ala'2-GIP-GluPal (A) and Val(8)GLP-1-GluPal (V) treatment resulted in an 1.7-fold ( $\mathrm{p}<0.05)$, 2-fold $(\mathrm{p}<0.01), 1.9$ fold $(\mathrm{p}<0.01), 1.8$-fold $(\mathrm{p}<0.01)$ and 1.9-fold $(\mathrm{p}<0.01)$ increase in $\mathrm{pBcl}-2$ levels, respectively, when compared to the stressed condition (Fig 5b). Although Exendin-4 (E) post-treatment upregulated the pBcl-2 level compared to control, it did not induce a significant rise as the other incretin analogues did.

\section{Discussion}

The results obtained in this study demonstrate plausible underlying mechanisms that forms the basis for enhanced cell survival exhibited by the incretin post-treatment of Rotenone-stressed SH-SY5Y cells. Interestingly, we found that cell proliferation as well as levels of key signalling markers increased with the Rotenone exposure. The BrdU assay was conducted on multiple experiments to confirm the unexpected findings of increased proliferation in stressed state. However, there are studies that indicate that at very low doses, Rotenone exhibits proliferative property. A few in vivo studies suggest that the experimental conditions control the selectivity of the rotenone insult (Hoglinger et al. 2003, Zhu et al. 2004, Meurers et al. 2009). The cell type as well as the duration and nature of the initial stress stimulus govern whether the cellular stress will trigger survival or death signalling (Fulda et al. 2010). In the current experimental setting, we observed enhanced proliferation at all three doses of Rotenone tested. Moreover, low level toxins could enhance cell proliferation as a protective reaction of the cell. In a recent study, Kim et al. found that reactive oxygen species (ROS) generation (from rotenone as one of the 
sources) upregulated the microRNA-210 expression resulting in increased proliferation of adipose-derived stem cells (Kim et al. 2013).

One of the mechanisms by which Akt gets activated is phosphorylation within the carboxy terminus at Ser473. Akt primarily promotes cell survival by inhibiting apoptosis involving phosphorylation and inactivation of targets like Bad (pro-apoptotic Bcl-2 family member) and forkhead transcription factors (Brunet et al. 1999, Yamaguchi \& Wang 2001). Therefore, we evaluated the levels of phospho-Akt (Ser473), pro-survival phospho-Bcl-2 (Ser70), pro-apoptotic phospho-BAD (Ser112) and phospho-Fox01 (Ser256). When under the stress, initially internal cellular pathways try to compensate for the abnormal condition but the cell starts dying when unable to cope with the prolonged stress. Having said that, the increase in pAkt (Ser473)/panAkt observed in the Rotenone stressed cells could be one of the ways the cells respond under stress. GLP-1/GIP Dual agonist post-treatment resulted in almost 3-fold increase $(\mathrm{p}<0.001)$ in the pAkt (Ser473)/panAkt expression when compared to control cells. This is in line with a recent study where dual GLP-1 and GIP receptor agonist increased the Akt expression in the MPTP mouse model of PD (Ji et al. 2015). Interestingly, we found significant increase in pAkt levels when treated with D-Ala ${ }^{2}-$ GIP-GluPal as compared to the Rotenone stressed untreated cells. In a previous study, we found a significant increase $(p<0.01)$ in the expression of pAkt (Ser473) in the Liraglutide pre-treated SH-SY5Y cells, stressed with methyl glyoxal (Sharma et al. 2014). One study testing Liraglutide showed that it protects cardiomyocytes from high glucose-induced apoptosis by activating the Epac-1/Akt pathway (Wu et al. 2014). Research on Exendin-4 treatment revealed elevated phosphorylation of the pro-survival kinase Akt (Robinson et al. 2015) and its potential involvement in protecting pancreatic beta cells from human islet amyloid polypeptideinduced cell damage (Fan et al. 2010). We too observed an increase in the pAkt (Ser473)/panAkt expression in the Exendin-4 post-treated cells (Fig 3a), which is in agreement with other studies.

As mitochondrial dysfunction forms key hallmark of PD and rotenone blocks complex I of the electron transport chain (ETC) in the inner mitochondrial membrane resulting in oxidative stress (Lin \& Beal 2006), we looked at the post-treatment effect of incretin 
analogues on the expression levels of mitochondrial proteins. Moreover, drugs that improve on mitochondrial damage might be useful as a PD treatment (Luo et al. 2015). Complex I is one of the main sites where premature electron leakage occurs leading to the superoxide $\left(\mathrm{O}_{2}^{-}\right)$production (West et al. 2011). Zhou et al. demonstrated that Exendin-4 pretreatment upregulates the levels of superoxide dismutase and preserves the mitochondrial membrane potential (Zhou et al. 2014a). Recent research has shown that GLP-1R stimulation ameliorates the detrimental cellular changes induced by oxidative stress. GLP-1 and Exendin-4 dose-dependently protect SH-SY5Y cells from hydrogen peroxide-induced stress ( $\mathrm{Li}$ et al. 2009, Li et al. 2010, Salcedo et al. 2012). Fan et al. investigated the effect of Exendin-4 treatment on mitochondrial function and found enhanced mitochondrial biogenesis (Fan et al. 2010). A recent proteomic study revealed potential protective roles of GLP-1 at the mitochondrial level (Ciregia et al. 2015). Our results support the finding that Liraglutide attenuates the effects of mitochondrial membrane damage in pancreatic $\beta$ cells (Ogata et al. 2014). Dihydrolipoamide acetyltransferase and lipoamide dehydrogenase along with Pyruvate dehydrogenase forms the pyruvate dehydrogenase (PDH) complex. The PDH complex is a nuclearencoded mitochondrial multi-enzyme complex that converts pyruvate to acetyl-CoA and $\mathrm{CO}_{2}$, the primary link between glycolysis and the tricarboxylic acid (TCA) cycle (Karlberg \& Andersson 2003) (Fig 6). PDHA1 plays a key role in the function of the PDH complex (Strumilo 2005). Further research results support the hypothesis that incretin-derived cAMP activates mTOR by mobilising intracellular $\mathrm{Ca}^{2+}$ stores that further upregulate mitochondrial dehydrogenases and result in enhanced ATP production (Kwon et al. 2004).

Autophagy and apoptosis are the two basic processes contributing to the maintenance of cellular homeostasis (Ghavami et al. 2014). Recent studies have found a key role for autophagy in neurodegenerative disorders, and it has been observed that autophagy is deregulated in PD brains (Cheung \& Ip 2009). Autophagy eliminates damaged organelles and protein aggregates. Therefore, therapeutic strategies to modulate the autophagy could be of great potential (Nixon 2013, Ghavami et al. 2014). To evaluate the role of incretin analogues in modulating the expression of autophagy-related genes (Atg) and other autophagy-associated markers, we analysed key biomarkers. Beclin-1 plays a central role 
in autophagy and it remains inactive when bound to the Bcl-2 but phosphorylation of Bcl2 disrupts this interaction and releases Beclin-1, thereby initiating autophagy (Kang et al. 2011). There are several core Atg forms that function in two ubiquitin-like conjugation systems essential for autophagosome formation. Firstly, we examined Atg7, the ubiquitin E1-like enzyme that activates Atg12 and mediates the formation of the autophagosome (Mizushima et al. 2011, Murrow \& Debnath 2015). Atg3, the E2-like enzyme is required in the second ubiquitin-like conjugation system essential for autophagosome formation (Murrow \& Debnath 2015). There are two schools of thought that describe (1) increase or (2) decrease in the autophagy under the effect of GLP-1 analogues. Our findings of increased Atg 7 supports the first school of thought. One report on the use of incretin therapy in beta cell-specific autophagy-deficient mice developed by Atg7 deletion (Atg7 ${ }^{\Delta \beta}$ cell) revealed that glucose-stimulated cAMP production was impaired in the autophagydeficient islets exposed to Exendin-4 (Kim et al. 2016). Another study on Liraglutide found that increase in autophagy plays a role in reducing the lipid accumulation in hepatic steatotic L- 02 cells (Zhou et al. 2014b). Both Exendin-4 and Liraglutide reduce hepatocyte steatosis and improve survival by promoting macro-autophagy (Sharma et al. 2011). Another study found found that Liraglutide prevents high glucose level induced insulinoma (INS-1) cell apoptosis by increasing autophagy (Chen et al. 2013). On the contrary, Zhao and co-workers examined the renoprotective effect of Liraglutide in the HK-2 cells and kidneys of diabetic rats and disclosed that Liraglutide inhibits autophagy (Zhao et al. 2015). It is crucial to maintain a proper level of autophagy in order to minimise levels of abnormal protein aggregates. Autophagy modulation is a potential therapeutic target and novel therapeutic agents that could improve autophagic activity or maintain mitochondrial homeostasis may reduce neuronal loss and slow down disease progression (Lynch-Day et al. 2012, Rubinsztein et al. 2012).

We confirmed the involvement of PI3K - mediated Akt activation by use of the PI3k inhibitor LY294002 and further show the possible pathways that lead to increased cell survival, autophagy and improved mitochondrial function. In addition, we present novel results comparing different incretin analogues. At the lowest dose of $1 \mathrm{nM}$, the novel GLP-1/GIP dual agonist increased the cell viability most efficiently among all six analogues tested. Finan et al. demonstrated enhanced anti-hyperglycemic and 
insulinotropic efficacy of GLP-1/GIP dual agonists relative to the currently best in class GLP-1 agonist liraglutide. The effective dose of the dual agonist was far lower compared to liraglutide, a finding that we confirmed in this in vitro study (Finan et al. 2013). Furthermore, at a dose of 100nM, Liraglutide, D-Ser2-Oxyntomodulin, a GLP-1/GIP Dual agonist and dAla(2)-GIP-GluPal improved cell survival significantly. In the BrdU assay, we found that 100nM Liraglutide enhanced cell proliferation the most among all the analogues. However, as this is an in vitro study, there are clear limitations, in particular, the pharmacodynamics in vivo will vary due to the different survival times of each analogue in the blood stream. Exendin-4 has a short survival time whereas the lipidated analogues such as liraglutide have a much longer survival time in the blood (Finan et al., 2013).

Incretin mimetics have shown neuroprotective effects in a range of animal models of disease, such as PD, AD, stroke and ALS (Holscher, 2014a). Importantly, GLP-1 analogues are currently in clinical trials, and have shown first good results in pilot study of PD with exendin-4 (Aviles-Olmos et al. 2013, 2014), and also in a pilot study of AD, showing robust protection in ${ }^{18}$ FDG-PET brain imaging by liraglutide (Gejl et al., 2016). Based on this success, phase II clinical trials in $\mathrm{AD}$ and PD are ongoing. Our findings presented in this paper shed light on some of the biochemical pathways and processes that may underlie the neuroprotective effects of the incretin hormones.

\section{Acknowledgement/conflict of Interest}

The work has been supported by grants from the Cure Parkinson's Trust (CPT) and the Rosetrees Trust. The authors JJ and MKS do not declare a conflict of interest. CH is a named inventor on several patents that name GLP-1 analogues or GIP analogues as novel treatments for AD or PD. The patents and patents applications are owned by Ulster University and Lancaster University. 


\section{Figure Legends}

Figure 1. Incretins enhance cell viability of rotenone stressed SH-SY5Y cells. $12 \mathrm{hrs}$ of post-treatment with $100 \mathrm{nM}$ incretin analogues against Rotenone stress $(0.1,1,10 \mu \mathrm{M})$ for $12 \mathrm{hrs}$ increases (a) cell survival, (b) decreases lactate dehydrogenase (LDH) levels of SH-SY5Y cells. From the initial experiments $1 \mu \mathrm{M}$ concentration of rotenone was decided for further studies. The effect of post-treatment with $1 \mathrm{nM}$ incretin analogues against $1 \mu \mathrm{M}$ rotenone stress was examined on the (c) cell viability (d) cytotoxicity of SH-SY5Y cells. Data are presented as mean \pm SEM and as a percentage of control. Statistical analysis was done by one-way ANOVA followed by Bonferroni’s Multiple Comparison test $(* p<$ $0.05, * * \mathrm{p}<0.01, * * * \mathrm{p}<0.001, * * * * \mathrm{p}<0.0001, \mathrm{~ns}$ : non-significant $), \mathrm{n}=5$.

Figure 2. Post-treatment with 10nM and 100nM Incretin analogues enhances cell survival and decreases cytotoxicity. The effect of post-treatment with 10nM incretin analogues against $1 \mu \mathrm{M}$ rotenone stress for $12 \mathrm{hrs}$ was examined on the (a) cell viability (b) cytotoxicity of SH-SY5Y cells. Thereafter, the effect of post-treatment with $100 \mathrm{nM}$ incretin analogues against $1 \mu \mathrm{M}$ rotenone stress was studied on the (c) cell survival (d) LDH levels. Data are presented as mean \pm SEM and as a percentage of control. Statistical analysis was done by one-way ANOVA followed by Bonferroni's Multiple Comparison test $(* \mathrm{p}<0.05, * * \mathrm{p}<0.01, * * * \mathrm{p}<0.001, * * * * \mathrm{p}<0.0001, \mathrm{~ns}$ : non-significant $), \mathrm{n}=5$.

Figure 3. Incretin analogues activate cell survival mechanism. Western blot analysis of the cells challenged with $1 \mu \mathrm{M}$ rotenone stress for $12 \mathrm{hrs}$ followed by a $12 \mathrm{hr}$ posttreatment with 100nM incretin analogues. There was an increase in the expression of (a) pAkt (Ser473)/panAkt, (b) pBcl-2 (Ser70), (c) phospho-BAD when compared to the control. Beta-actin was used as an internal loading control. Results are expressed as mean \pm SEM of three independent experiments as a percentage of control. Statistical analysis was by one-way ANOVA followed by Bonferroni's Multiple Comparison test $(* p<0.05$, $* * \mathrm{p}<0.01, * * * \mathrm{p}<0.001, * * * * \mathrm{p}<0.0001, \mathrm{~ns}$ : non-significant).

Figure 4. Incretin analogues protect against mitochondrial damage and autophagy 
impairment induced by rotenone stress. Western blot analysis of the cells exposed to $1 \mu \mathrm{M}$ rotenone stress for $12 \mathrm{hrs}$ followed by post-treatment with $100 \mathrm{nM}$ incretin analogues for $12 \mathrm{hrs}$. There was an increase in the expression of (a) PDH (pyruvate dehydrogenase), and (b) an increase in the expression of autophagy associated marker protein Atg7. the Beta-actin was used as an internal loading control. Results are expressed as mean \pm SEM of three independent experiments as a percentage of control. Statistical analysis by oneway ANOVA followed by Bonferroni's Multiple Comparison test $\left(* * \mathrm{p}<0.01,{ }^{* * *} \mathrm{p}<\right.$ $0.001, * * *$ p $<0.0001)$.

\section{Figure 5. Incretin analogues ameliorate the inhibitory effect of LY294002 on} autophagy and cell survival. SH-SY5Y cells were exposed to 50 $\mu$ M LY294002, PI3K inhibitor for $1 \mathrm{hr}$ and post-treated with 100nM incretin analogues for $1 \mathrm{hr}$. Western blot analysis shows that there was a significant decrease in the expression of (a) $\operatorname{Atg} 3$ in the untreated state that was ameliorated by the post-treatment with incretin analogues, (b) pBcl-2 expression was enhanced by incretin analogues. GAPDH was used as an internal loading control. Results are expressed as mean \pm SEM of three independent experiments as a percentage of control. Statistical analysis was by one-way ANOVA followed by Bonferroni's Multiple Comparison test $\left({ }^{*} \mathrm{p}<0.05,{ }^{* *} \mathrm{p}<0.01,{ }^{* * *} \mathrm{p}<0.001, * * * * \mathrm{p}<\right.$ 0.0001, ns: non-significant).

\section{Figure 6. Potential mechanism underlying the neuroprotection exhibited by Incretin} analogues against Rotenone stress. The diagrammatic representation of the effect of post-treatment with Incretin analogues against Rotenone stress summarises different events involved in the cellular signalling. The protective effect of incretin analogues in the SH-SY5Y human neuroblastoma cells against the Rotenone toxicity is mediated by PI3K/Akt pathway. When Incretin analogue binds to the GPCR GLP-1 or GIP receptor, it activates PI3K resulting in phosphorylation of Akt at site Ser473. Akt activation results in 1. Increase in autophagy by releasing Beclin-1 from Bcl-2, 2. Increase in survival by inhibition of apoptosis - phosphorylation of $\mathrm{Bcl}-2$ that prevents cytochrome $\mathrm{c}$ release from inner mitochondrial space (IMS), 3. Phosphorylation and inactivation of proapoptotic Bad and transcription factor Foxo1. Post-treatment with incretin analogues 
ameliorate mitochondrial dysfunctioning by 1 . an increased expression of (cytochrome $\mathrm{c}$ oxidase) Cox IV and (superoxide dismutase) SOD1, 2. Increase in (heat shock protein 60) HSP60 and Prohibitins (PHB1), 3. Increased Pyruvate dehydrogenase (PDH) expression. Abbrev.: GLP-1R = glucose-dependent insulinotropic peptide receptor; GIPR = glucosedependent insulinotropic peptide receptor; PI3K = phosphoinositide 3 kinase; OMM = outer mitochondrial membrane; IMM = inner mitochondrial membrane; IMS = inter membrane space; $\mathrm{Bcl}-2$ = B-cell lymphoma 2; $\mathrm{Bad}=\mathrm{Bcl}$-2-associated death promoter; SOD1 = superoxide dismutase; $\mathrm{PDH}=$ pyruvate dehydrogenase; Atg7 = Autophagyrelated protein 7; $\operatorname{Atg} 3=$ Autophagy-related protein 3 . 


\section{References}

Alberio, T., Lopiano, L. and Fasano, M. (2012) Cellular models to investigate biochemical pathways in Parkinson's disease. FEBS J, 279, 1146-1155.

Andreu, C. I., Woehlbier, U., Torres, M. and Hetz, C. (2012) Protein disulfide isomerases in neurodegeneration: from disease mechanisms to biomedical applications. FEBS Lett, 586, 2826-2834.

Aviles-Olmos, I., Dickson, J., Kefalopoulou, Z. et al. (2014) Motor and cognitive advantages persist 12 months after exenatide exposure in Parkinson's disease. $J$ Parkinsons Dis, 4, 337-344.

Aviles-Olmos, I., Limousin, P., Lees, A. and Foltynie, T. (2013) Parkinson's disease, insulin resistance and novel agents of neuroprotection. Brain, 136, 374-384.

Bassil, F., Fernagut, P. O., Bezard, E. and Meissner, W. G. (2014) Insulin, IGF-1 and GLP-1 signaling in neurodegenerative disorders: targets for disease modification? Prog Neurobiol, 118, 1-18.

Bergeron, J. J., Brenner, M. B., Thomas, D. Y. and Williams, D. B. (1994) Calnexin: a membrane-bound chaperone of the endoplasmic reticulum. Trends Biochem Sci, 19, 124128.

Bertilsson, G., Patrone, C., Zachrisson, O. et al. (2008) Peptide hormone exendin-4 stimulates subventricular zone neurogenesis in the adult rodent brain and induces recovery in an animal model of Parkinson's disease. J Neurosci Res, 86, 326-338.

Brunet, A., Bonni, A., Zigmond, M. J. et al. (1999) Akt promotes cell survival by phosphorylating and inhibiting a Forkhead transcription factor. Cell, 96, 857-868.

Campbell, J. E. and Drucker, D. J. (2013) Pharmacology, physiology, and mechanisms of incretin hormone action. Cell Metab, 17, 819-837.

Cao L., Li D., Feng P., Li L., Xue GF, Li G., Hölscher C.(2016): A novel dual GLP-1 and GIP incretin receptor agonist is neuroprotective in a mouse model of Parkinson's disease by reducing chronic inflammation in the brain. Neuroreport, in press.

Chen, Z. F., Li, Y. B., Han, J. Y., Yin, J. J., Wang, Y., Zhu, L. B. and Xie, G. Y. (2013) Liraglutide prevents high glucose level induced insulinoma cells apoptosis by targeting autophagy. Chin Med J (Engl), 126, 937-941. 
Cheung, Z. H. and Ip, N. Y. (2009) The emerging role of autophagy in Parkinson's disease. Mol Brain, 2, 29.

Cho, Y. M., Fujita, Y. and Kieffer, T. J. (2014) Glucagon-like peptide-1: glucose homeostasis and beyond. Annu Rev Physiol, 76, 535-559.

Ciregia, F., Giusti, L., Ronci, M. et al. (2015) Glucagon-like peptide 1 protects INS-1E mitochondria against palmitate-mediated beta-cell dysfunction: a proteomic study. $\mathrm{Mol}$ Biosyst, 11, 1696-1707.

Faivre, E. and Holscher, C. (2013) D-Ala2GIP facilitated synaptic plasticity and reduces plaque load in aged wild type mice and in an Alzheimer's disease mouse model. $J$ Alzheimers Dis, 35, 267-283.

Fan, R., Li, X., Gu, X., Chan, J. C. and Xu, G. (2010) Exendin-4 protects pancreatic beta cells from human islet amyloid polypeptide-induced cell damage: potential involvement of AKT and mitochondria biogenesis. Diabetes Obes Metab, 12, 815-824.

Finan, B., Ma, T., Ottaway, N. et al. (2013) Unimolecular dual incretins maximize metabolic benefits in rodents, monkeys, and humans. Sci Transl Med, 5, 209ra151.

Fulda, S., Gorman, A. M., Hori, O. and Samali, A. (2010) Cellular stress responses: cell survival and cell death. Int J Cell Biol, 2010, 214074.

Gengler, S., McClean, P. L., McCurtin, R., Gault, V. A. and Holscher, C. (2012) $\mathrm{Val}(8)$ GLP-1 rescues synaptic plasticity and reduces dense core plaques in APP/PS1 mice. Neurobiol Aging, 33, 265-276.

Gejl M, Gjedde A, Egefjord L, Møller A, Hansen SB, Vang K, Rodell AB, Braendgaard H, Gottrup H, Schacht A, Møller N, Brock B, Rungby J (2016) In Alzheimer's Disease, Six-Month Treatment with GLP-1 Analogue Prevents Decline of Brain Glucose Metabolism: Randomized, Placebo-Controlled, Double-Blind Clinical Trial. Frontiers Aging Neurosci, 8, 1-10.

Ghavami, S., Shojaei, S., Yeganeh, B. et al. (2014) Autophagy and apoptosis dysfunction in neurodegenerative disorders. Prog Neurobiol, 112, 24-49.

Harkavyi, A., Abuirmeileh, A., Lever, R., Kingsbury, A. E., Biggs, C. S. and Whitton, P. S. (2008) Glucagon-like peptide 1 receptor stimulation reverses key deficits in distinct rodent models of Parkinson's disease. J Neuroinflammation, 5, 19.

Hoglinger, G. U., Feger, J., Prigent, A., Michel, P. P., Parain, K., Champy, P., Ruberg, 
M., Oertel, W. H. and Hirsch, E. C. (2003) Chronic systemic complex I inhibition induces a hypokinetic multisystem degeneration in rats. J Neurochem, 84, 491-502.

Holscher, C. (2012) Potential role of glucagon-like peptide-1 (GLP-1) in neuroprotection. CNS Drugs, 26, 871-882.

Holscher, C. (2014a) Central effects of GLP-1: new opportunities for treatments of neurodegenerative diseases. J Endocrinol, 221, T31-41.

Holscher, C. (2014b) New drug treatments show neuroprotective effects in Alzheimer's and Parkinson's diseases. Neural Regen Res, 9, 1870-1873.

Hu, G., Jousilahti, P., Bidel, S., Antikainen, R. and Tuomilehto, J. (2007) Type 2 diabetes and the risk of Parkinson's disease. Diabetes Care, 30, 842-847.

Ji, C., Xue, G. F., Lijun, C., Feng, P., Li, D., Li, L., Li, G. and Holscher, C. (2015) A novel dual GLP-1 and GIP receptor agonist is neuroprotective in the MPTP mouse model of Parkinson's disease by increasing expression of BNDF. Brain Res.

Johansen, T. and Lamark, T. (2011) Selective autophagy mediated by autophagic adapter proteins. Autophagy, 7, 279-296.

Kang, R., Zeh, H. J., Lotze, M. T. and Tang, D. (2011) The Beclin 1 network regulates autophagy and apoptosis. Cell Death Differ, 18, 571-580.

Karlberg, E. O. and Andersson, S. G. (2003) Mitochondrial gene history and mRNA localization: is there a correlation? Nat Rev Genet, 4, 391-397.

Keane, P. C., Kurzawa, M., Blain, P. G. and Morris, C. M. (2011) Mitochondrial dysfunction in Parkinson's disease. Parkinsons Dis, 2011, 716871.

Kim, J. H., Park, S. G., Song, S. Y., Kim, J. K. and Sung, J. H. (2013) Reactive oxygen species-responsive miR-210 regulates proliferation and migration of adipose-derived stem cells via PTPN2. Cell Death Dis, 4, e588.

Kim, M. J., Choi, O. K., Chae, K. S. et al. (2016) Autophagy deficiency in beta cells blunts incretin-induced suppression of glucagon release from alpha cells. Islets, 0 .

Kim, S., Moon, M. and Park, S. (2009) Exendin-4 protects dopaminergic neurons by inhibition of microglial activation and matrix metalloproteinase- 3 expression in an animal model of Parkinson's disease. J Endocrinol, 202, 431-439.

Kuang, X. L., Liu, F., Chen, H. et al. (2014) Reductions of the components of the calreticulin/calnexin quality-control system by proteasome inhibitors and their relevance 
in a rodent model of Parkinson's disease. J Neurosci Res, 92, 1319-1329.

Kwon, G., Marshall, C. A., Pappan, K. L., Remedi, M. S. and McDaniel, M. L. (2004) Signaling elements involved in the metabolic regulation of mTOR by nutrients, incretins, and growth factors in islets. Diabetes, 53 Suppl 3, S225-232.

Lennox, R., Porter, D. W., Flatt, P. R. and Gault, V. A. (2013) (Val(8))GLP-1-Glu-PAL: a GLP-1 agonist that improves hippocampal neurogenesis, glucose homeostasis, and beta-cell function in high-fat-fed mice. ChemMedChem, 8, 595-602.

Li, Y., Liu, W., Li, L. and Holscher, C. (2016) Neuroprotective effects of a GIP analogue in the MPTP Parkinson's disease mouse model. Neuropharmacology, 101, 255-263.

Li, Y., Perry, T., Kindy, M. S. et al. (2009) GLP-1 receptor stimulation preserves primary cortical and dopaminergic neurons in cellular and rodent models of stroke and Parkinsonism. Proc Natl Acad Sci U S A, 106, 1285-1290.

Li, Y., Tweedie, D., Mattson, M. P., Holloway, H. W. and Greig, N. H. (2010) Enhancing the GLP-1 receptor signaling pathway leads to proliferation and neuroprotection in human neuroblastoma cells. J Neurochem, 113, 1621-1631.

Lin, M. T. and Beal, M. F. (2006) Mitochondrial dysfunction and oxidative stress in neurodegenerative diseases. Nature, 443, 787-795.

Liu, W., Jalewa, J., Sharma, M., Li, G., Li, L. and Holscher, C. (2015a) Neuroprotective effects of lixisenatide and liraglutide in the 1-methyl-4-phenyl-1,2,3,6-tetrahydropyridine mouse model of Parkinson's disease. Neuroscience, 303, 42-50.

Liu, W., Li, Y., Jalewa, J., Saunders-Wood, T., Li, L. and Holscher, C. (2015b) Neuroprotective effects of an oxyntomodulin analogue in the MPTP mouse model of Parkinson's disease. Eur J Pharmacol, 765, 284-290.

Luo, Y., Hoffer, A., Hoffer, B. and Qi, X. (2015) Mitochondria: A Therapeutic Target for Parkinson's Disease? Int J Mol Sci, 16, 20704-20730.

Lynch-Day, M. A., Mao, K., Wang, K., Zhao, M. and Klionsky, D. J. (2012) The role of autophagy in Parkinson's disease. Cold Spring Harb Perspect Med, 2, a009357.

Manandhar, B. and Ahn, J. M. (2015) Glucagon-like peptide-1 (GLP-1) analogs: recent advances, new possibilities, and therapeutic implications. J Med Chem, 58, 1020-1037. Martin, C. M., Irwin, N., Flatt, P. R. and Gault, V. A. (2013) A novel acylated form of (dAla(2))GIP with improved antidiabetic potential, lacking effect on body fat stores. 
Biochim Biophys Acta, 1830, 3407-3413.

McClean, P. L. and Holscher, C. (2014) Liraglutide can reverse memory impairment, synaptic loss and reduce plaque load in aged APP/PS1 mice, a model of Alzheimer's disease. Neuropharmacology, 76 Pt A, 57-67.

McClean, P. L., Parthsarathy, V., Faivre, E. and Holscher, C. (2011) The diabetes drug liraglutide prevents degenerative processes in a mouse model of Alzheimer's disease. $J$ Neurosci, 31, 6587-6594.

Merkwirth, C. and Langer, T. (2009) Prohibitin function within mitochondria: essential roles for cell proliferation and cristae morphogenesis. Biochim Biophys Acta, 1793, 2732.

Meurers, B. H., Zhu, C., Fernagut, P. O. et al. (2009) Low dose rotenone treatment causes selective transcriptional activation of cell death related pathways in dopaminergic neurons in vivo. Neurobiol Dis, 33, 182-192.

Mizushima, N., Yoshimori, T. and Ohsumi, Y. (2011) The role of Atg proteins in autophagosome formation. Annu Rev Cell Dev Biol, 27, 107-132.

Murrow, L. and Debnath, J. (2015) ATG12-ATG3 connects basal autophagy and late endosome function. Autophagy, 11, 961-962.

Nixon, R. A. (2013) The role of autophagy in neurodegenerative disease. Nat Med, 19, 983-997.

Obeso, J. A., Rodriguez-Oroz, M. C., Goetz, C. G. et al. (2010) Missing pieces in the Parkinson's disease puzzle. Nat Med, 16, 653-661.

Ogata, M., Iwasaki, N., Ide, R., Takizawa, M. and Uchigata, Y. (2014) GLP-1-related proteins attenuate the effects of mitochondrial membrane damage in pancreatic beta cells. Biochem Biophys Res Commun, 447, 133-138.

Pocai, A. (2012) Unraveling oxyntomodulin, GLP1's enigmatic brother. J Endocrinol, 215, 335-346.

Robinson, E., Cassidy, R. S., Tate, M. et al. (2015) Exendin-4 protects against postmyocardial infarction remodelling via specific actions on inflammation and the extracellular matrix. Basic Res Cardiol, 110, 20.

Rowland, A. A. and Voeltz, G. K. (2012) Endoplasmic reticulum-mitochondria contacts: function of the junction. Nat Rev Mol Cell Biol, 13, 607-625. 
Rubinsztein, D. C., Codogno, P. and Levine, B. (2012) Autophagy modulation as a potential therapeutic target for diverse diseases. Nat Rev Drug Discov, 11, 709-730.

Rugarli, E. I. and Langer, T. (2012) Mitochondrial quality control: a matter of life and death for neurons. EMBO J, 31, 1336-1349.

Salcedo, I., Tweedie, D., Li, Y. and Greig, N. H. (2012) Neuroprotective and neurotrophic actions of glucagon-like peptide-1: an emerging opportunity to treat neurodegenerative and cerebrovascular disorders. Br J Pharmacol, 166, 1586-1599.

Schapira, A. H. and Jenner, P. (2011) Etiology and pathogenesis of Parkinson's disease. Mov Disord, 26, 1049-1055.

Schisano, B., Harte, A. L., Lois, K. et al. (2012) GLP-1 analogue, Liraglutide protects human umbilical vein endothelial cells against high glucose induced endoplasmic reticulum stress. Regul Pept, 174, 46-52.

Sharma, M. K., Jalewa, J. and Holscher, C. (2014) Neuroprotective and anti-apoptotic effects of liraglutide on SH-SY5Y cells exposed to methylglyoxal stress. J Neurochem, 128, 459-471.

Sharma, S., Mells, J. E., Fu, P. P., Saxena, N. K. and Anania, F. A. (2011) GLP-1 analogs reduce hepatocyte steatosis and improve survival by enhancing the unfolded protein response and promoting macroautophagy. PLoS One, 6, e25269.

Shulman, J. M., De Jager, P. L. and Feany, M. B. (2011) Parkinson's disease: genetics and pathogenesis. Annu Rev Pathol, 6, 193-222.

Strumilo, S. (2005) Short-term regulation of the mammalian pyruvate dehydrogenase complex. Acta Biochim Pol, 52, 759-764.

West, A. P., Shadel, G. S. and Ghosh, S. (2011) Mitochondria in innate immune responses. Nat Rev Immunol, 11, 389-402.

Williams, D. B. (2006) Beyond lectins: the calnexin/calreticulin chaperone system of the endoplasmic reticulum. J Cell Sci, 119, 615-623.

Wu, X. M., Ou, Q. Y., Zhao, W., Liu, J. and Zhang, H. (2014) The GLP-1 analogue liraglutide protects cardiomyocytes from high glucose-induced apoptosis by activating the Epac-1/Akt pathway. Exp Clin Endocrinol Diabetes, 122, 608-614.

Yamaguchi, H. and Wang, H. G. (2001) The protein kinase PKB/Akt regulates cell survival and apoptosis by inhibiting Bax conformational change. Oncogene, 20, 7779- 
7786.

Zhang, Y., Chen, Y., Li, L. and Holscher, C. (2015) Neuroprotective effects of (Val8)GLP-1-Glu-PAL in the MPTP Parkinson's disease mouse model. Behav Brain Res, 293, 107-113.

Zhao, X., Liu, G., Shen, H., Gao, B., Li, X., Fu, J., Zhou, J. and Ji, Q. (2015) Liraglutide inhibits autophagy and apoptosis induced by high glucose through GLP-1R in renal tubular epithelial cells. Int J Mol Med, 35, 684-692.

Zhou, H., Yang, J., Xin, T. et al. (2014a) Exendin-4 protects adipose-derived mesenchymal stem cells from apoptosis induced by hydrogen peroxide through the PI3K/Akt-Sfrp2 pathways. Free Radic Biol Med, 77, 363-375.

Zhou, S. W., Zhang, M. and Zhu, M. (2014b) Liraglutide reduces lipid accumulation in steatotic L02 cells by enhancing autophagy. Mol Med Rep, 10, 2351-2357.

Zhu, C., Vourc'h, P., Fernagut, P. O., Fleming, S. M., Lacan, S., Dicarlo, C. D., Seaman, R. L. and Chesselet, M. F. (2004) Variable effects of chronic subcutaneous administration of rotenone on striatal histology. J Comp Neurol, 478, 418-426. 
(a)

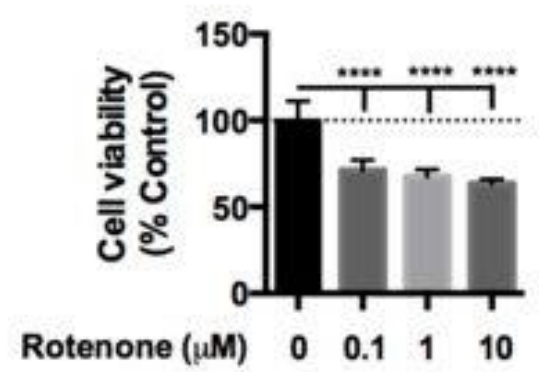

(b)

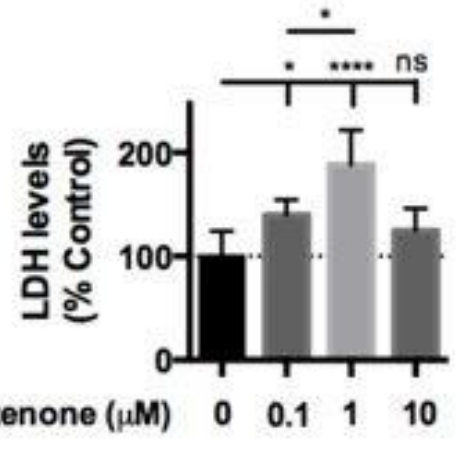

(c)

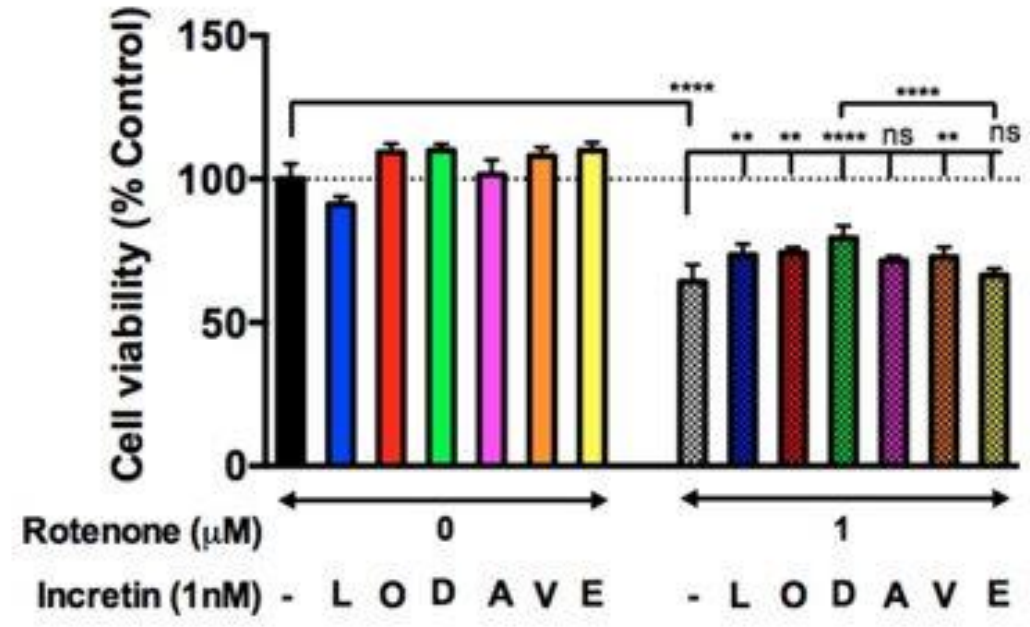

(d)

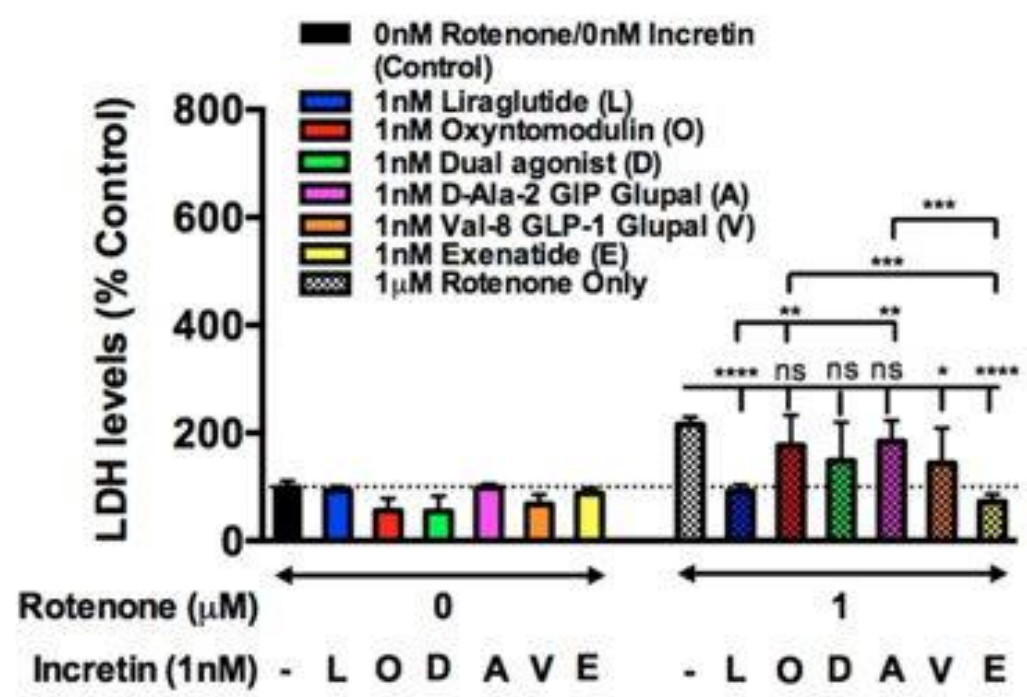

Fig 1 
(a)

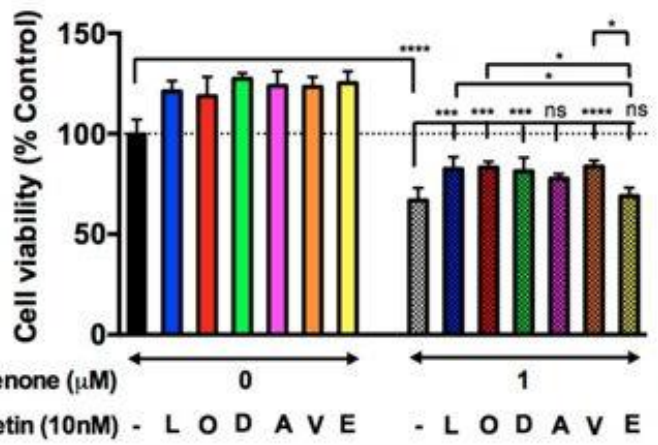

(c)

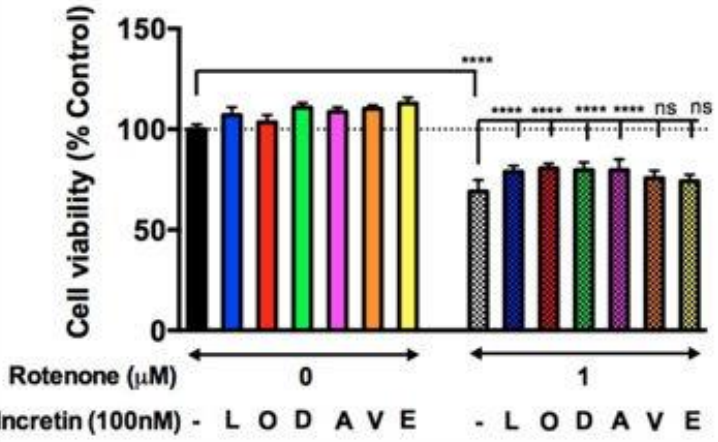

(b)

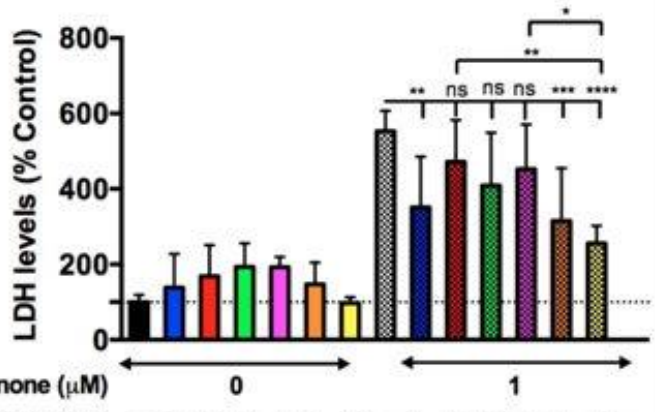

Incretin (10nM) - L O D A V E - L O D A V E

(d)

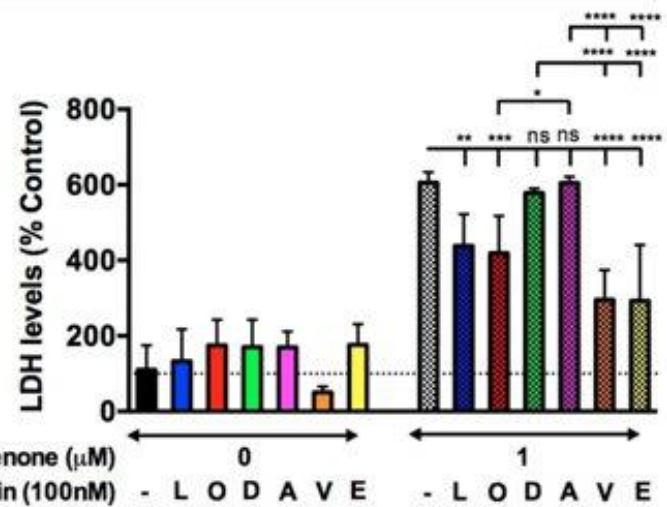

Fig. 2

(a)
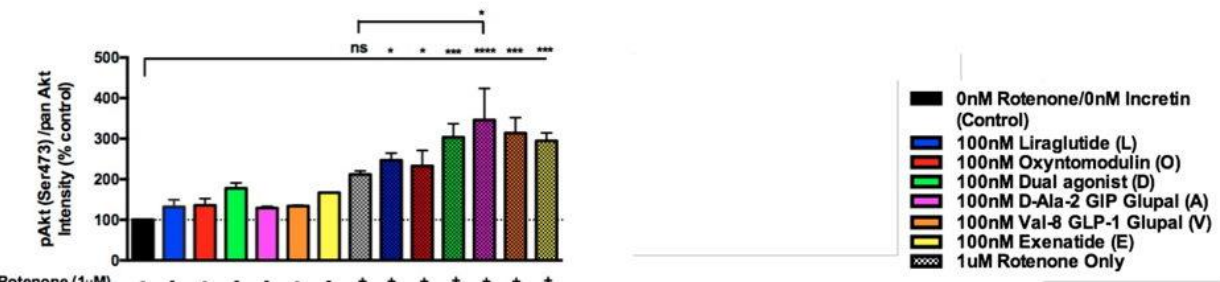

Rotenone $(1 \mathrm{\mu M})$. . . . . . + + + + +

Incretin (100nM) - L O D A V E - L O D A V E

M.W. kDa

60 - phospho Akt (Ser473)

60 -

(b)

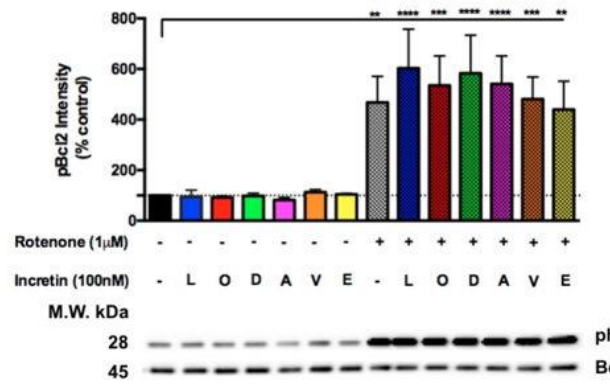

(c)

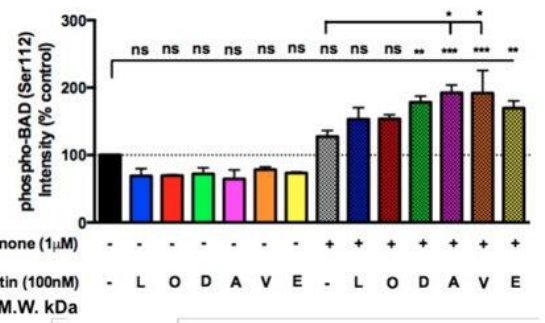

M.W. KDa

phospho-BAD (S112)

45

Fig. 3 
(a)

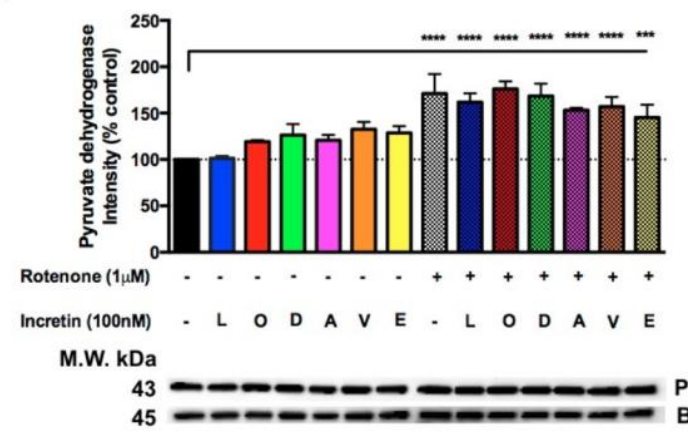

(b)

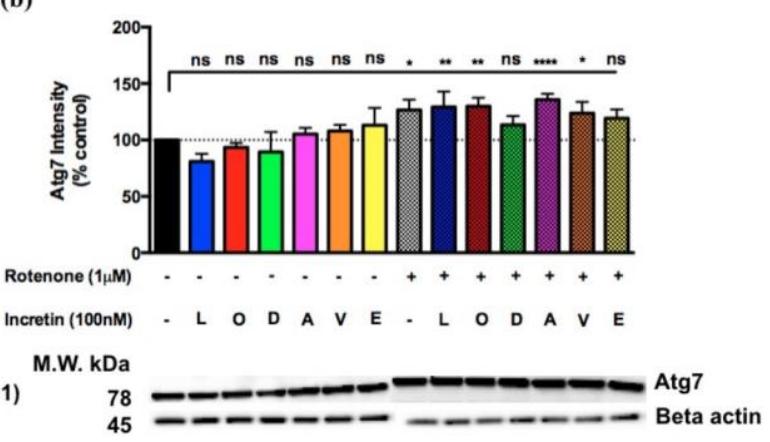

Fig. 4
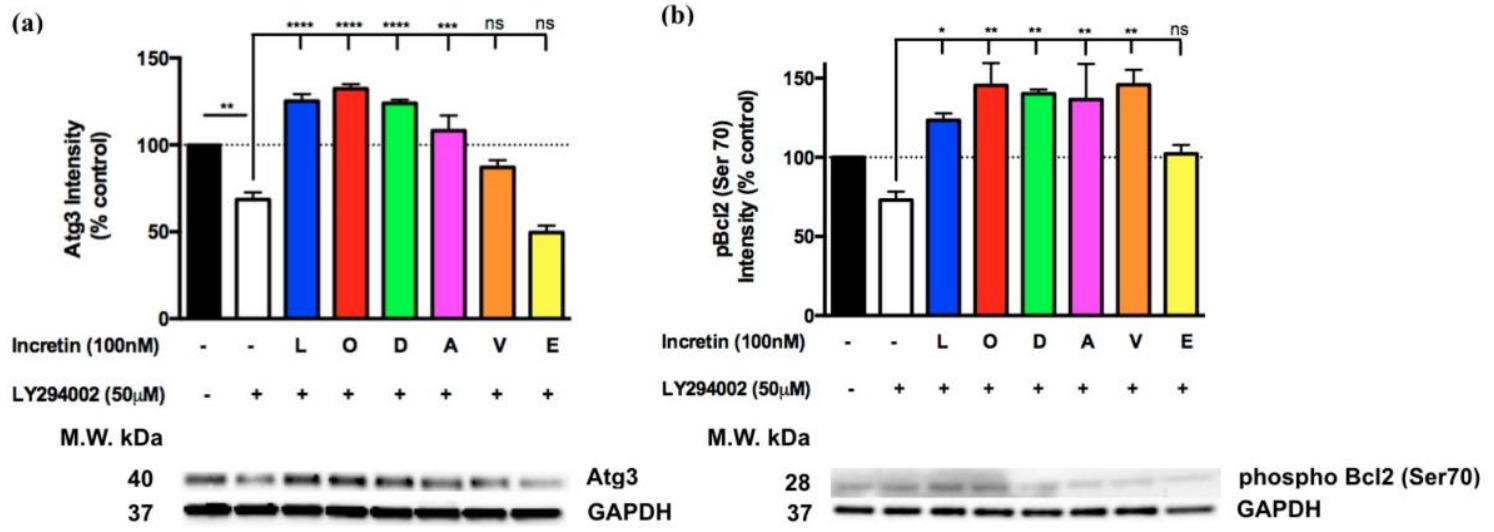

Fig. 5 


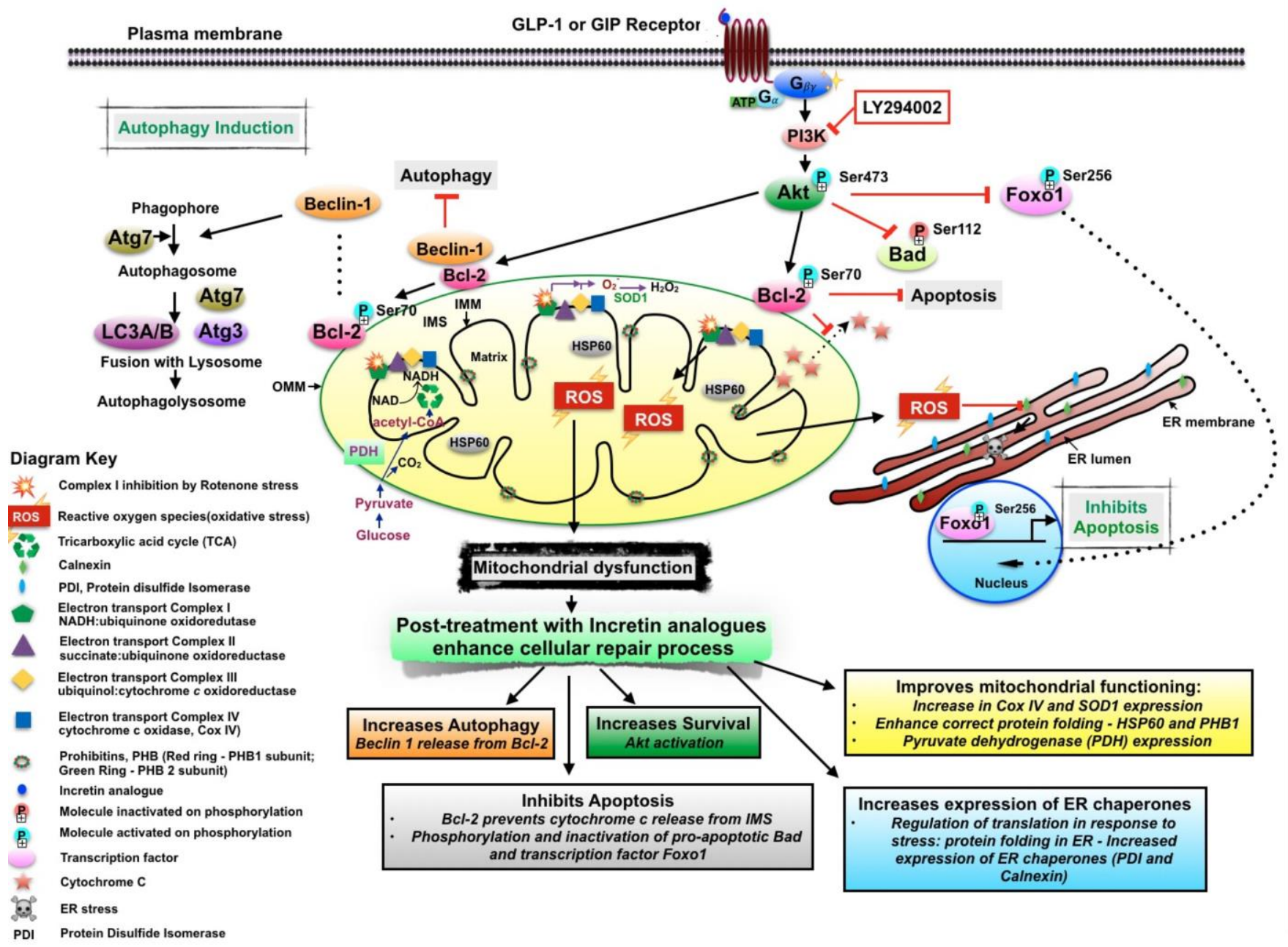

Fig. 6. 